Meta

Journal des traducteurs

Translators' Journal

\title{
Les indésirables
}

\section{Robert Dubuc}

Volume 12, numéro 2, juin 1967

URI : https://id.erudit.org/iderudit/002599ar

DOI : https://doi.org/10.7202/002599ar

Aller au sommaire du numéro

Éditeur(s)

Les Presses de l'Université de Montréal

ISSN

0026-0452 (imprimé)

1492-1421 (numérique)

Découvrir la revue

Citer ce document

Dubuc, R. (1967). Les indésirables. Meta, 12(2), 50-51.

https://doi.org/10.7202/002599ar

Ce document est protégé par la loi sur le droit d'auteur. L’utilisation des services d'Érudit (y compris la reproduction) est assujettie à sa politique d'utilisation que vous pouvez consulter en ligne.

https://apropos.erudit.org/fr/usagers/politique-dutilisation/
Cet article est diffusé et préservé par Érudit.

Érudit est un consortium interuniversitaire sans but lucratif composé de l'Université de Montréal, l'Université Laval et l'Université du Québec à Montréal. Il a pour mission la promotion et la valorisation de la recherche. https://www.erudit.org/fr/ 


\section{PROBLÈMES ET SOLUTIONS}

\section{LES INDÉSIRABLES}

On parle beaucoup aujourd'hui de la lutte contre les bruits nuisibles. On estime en effet que la prolifération du bruit ajoute à la tension de nos contemporains. Pendant que les spécialistes de l'acoustique luttent pour éliminer de nos vies les bruits nuisibles, l'industrie lance sur le marché quelque bruyante machine qui pourchasse le silence jusqu'en ses derniers retranchements. Les techniciens de la radio-télévision mènent à leur façon la guerre contre les bruits nuisibles: leur objectif n'est cependant pas de propager le silence, mais d'épurer le message à transmettre de tout bruit qui gêne l'audition. Il suffit de se rappeler les premières émissions radiophoniques des années trente pour constater les énormes progrès accomplis dans ce domaine. Les bruits nuisibles sont en effet légion.

Pour les profanes que nous sommes, on pourrait grouper sommairement les bruits nuisibles de la radiodiffusion en trois catégories: les pique-assiettes, les empiéteurs et les casse-pieds.

Les « pique-assiettes » sont les parasites de l'arbre généalogique des bruits. Le récepteur radiophonique en crache quelques-uns s'il lui faut capter quelque station éloignée. Ces bruits, d'origine atmosphérique, sont attribuables, paraît-il, aux variations de l'état électrique de l'atmosphère. C'est à tort que nous donnons à ces bruits l'appellation de «statique »' (de l'anglais static). On dit par exemple: «La présence de statique gêne très souvent la réception sur ondes courtes. » En français, ces trouble-fête portent le nom de parasites atmosphériques. C'est évidemment l'expression à retenir pour rendre l'anglais static.

Cousins des parasites atmosphériques, les parasites industriels (man-made interference) proviennent des perturbations que produisent dans les signaux radioélectriques les appareils et les installations électriques. Le rasage matinal, fait avec ces poivrières grondeuses qu'on qualifie de rasoirs, a pu fournir à chacun l'expérience concrète d'une forme de parasites industriels. Soulignons, en passant, que le qualificatif «industriel »s'applique aussi aux parasites d'origine domestique. Pour les techniciens, évidemment, seuls les parasites causés par l'industrie sont 
source véritable d'ennuis. Leur préoccupation a contribué à créer cette désignation. Mais, essentiellement, les troubles de réception causés par la proximité des moteurs d'une usine sont de même nature que ceux qu'occasionnent temporairement le rasoir électrique, le robot (electric mixer) et les autres appareils qui peuplent l'univers motorisé d'un foyer au $\mathrm{xx}^{\mathrm{e}}$ siècle.

On n'a pas cru devoir créer un nouveau terme pour désigner des bruits qui au fond sont semblables. Un tel effort aurait d'ailleurs été inutile. Abondance de biens nuit, dit-on. En linguistique, peut-être plus qu'ailleurs. Les grammairiens amateurs tendent trop souvent à négliger la part d'arbitraire inhérente à toute désignation. Pour qu'une désignation soit bonne, il faut et il suffit qu'elle s'applique sans ambiguïté à l'objet qu'elle nomme. Les étiquettes-définitions, en dépit de leur grande précision, sont souvent des créations mort-nées.

L'ensemble des bruits nuisibles attribuables à une source d'émission extérieure à la source utile porte le nom générique d'interference en anglais. Ici nous guette un faux ami. «Interférence» en français a beaucoup moins d'extension que son sosie anglais. Il y a interférence en français quand il y a perception de « signaux provenant de stations indésirables émettant sur une fréquence voisine $\gg^{1}$. Le plus souvent, il convient de rendre interference par parasites, sans qualificatif.

Les bruits empiéteurs comprennent, en plus de l'interférence, la diaphonie (cross-talk) et le brouillage (jamming). Mauvais coucheurs, ces bruits tendent toujours à s'emparer du domaine d'autrui. Dans le cas de l'interférence, ce sont les signaux d'une station indésirable qui viennent empiéter sur le domaine d'une autre; la diaphonie désigne un mélange de signaux à la réception, et le brouillage n'est en somme qu'une diaphonie artificielle dont le but est d'empêcher la réception d'un signal donné. C'est un jeu qu'on pratique souvent en temps de guerre.

Les bruits mécaniques ferment le cortège des bruits nuisibles. On a livré, avec passablemẹnt de succès d'ailleurs, une guerre sans merci à ces casse-pieds de la mise en ondes. Les principaux sont les bruits de fond (back-ground noises), tel ce souffle du haut-parleur perceptible en l'absence de signal sonore, et les bruits de surface ou d'aiguille (surface noises) que fait entendre le frottement de l'aiguille lorsque le disque commence à subir « des ans l'irréparable outrage ».

La famille des bruits m'en voudra-t-elle de lui avoir collé, pour les besoins de mon propos, des étiquettes qui servent ordinairement à désigner les individus plus ou moins désirables qui peuplent notre société ? Mais qu'a-t-elle à craindre pour son prestige? Au siècle de la musique concrète, tous les bruits sont ennoblis !

ROBERT DUBUC

\section{FAUT-IL RÉCLAMER?}

Dans notre pays bilingue, on traduit a cat par un chat, ce qui est bien; on traduit parfois a translator par un transcripteur, ce qui est parfois le cas; et dans les assurances, lorsqu'il faut rendre le mot claim, on dit « réclamation», ce qui est un calque de transcripteur. Un anglicisme tenace, qui a jusqu'à ce jour résisté aux condamnations, qui a la vie dure comme Raminagrobis lui-même. À tous moments

1. Jean Brun, Dictionnaire de la radio, Paris, Albin Michel, 1949, p. 286. 